\title{
TRATAMENTO AMBULATORIAL DA PROCTORRAGIA - IMPORTÂNCIA DA FOTOCOAGULAÇÃO COM RAIO INFRAVERMELHO
}

\author{
JOSÉ RIBAMAR BALDEZ ${ }^{I}$
}

${ }^{1}$ Clínica de Proctologia do Maranhão

Baldez JR. Tratamento Ambulatorial da Proctorragia - Importância da Fotocoagulação com Raio Infravermelho. Rev bras Coloproct, 2005;26(2):133-137.

RESUMO: Um grande número de pacientes são encaminhados ao Coloproctologista em virtude da ocorrência de sangramento retal. O sangramento retal vermelho vivo usualmente traduz a presença de uma patologia localizada até a flexura esplênica, sendo então necessária para a sua avaliação a realização de uma retossigmoidoscopia flexível. Durante esta propedêutica, na grande maioria dos pacientes, são encontradas Hemorróidas Internas, estabelecendo-se então o seguinte dilema: se realmente não existe outra causa mais séria da hemorragia e se temos a obrigação de tratar todas as Hemorróidas Internas encontradas como causa isolada do sintoma. Temos seguido a conduta de tratar todos os pacientes que apresentam episódios de sangramento retal devido a Hemorróidas. Eles têm a base de seus plexos hemorroidários internos coagulados com Raio Infravermelho. Dos 250 pacientes atendidos na Clínica de Proctologia do Maranhão em virtude da ocorrência de sangramento retal, 200 foram submetidos à fotocoagulação com Infravermelho, somente em uma sessão de tratamento. Concluímos que a fotocoagulação é um método simples e eficaz de tratamento, pode ser aplicado ao final de qualquer avaliação para sangramento retal rutilante devido a Hemorróidas Internas e que tal conduta possibilita que a maioria dos pacientes possam ser tratados em sua única consulta.

Descritores: Hemorragia retal; Hemorróidas; Fotocoagulação

\section{INTRODUÇÃO}

Muitos pacientes encaminhados para consulta coloproctológica por apresentarem hemorragia retal com sangue vermelho vivo, habitualmente sofrem de Hemorróidas. Sem dúvida, posto que padecer de Hemorróidas é quase universal, o achado de plexos hemorroidários congestionados não significa que estes sejam a causa da hemorragia. Por conseguinte, é necessário explorar minuciosamente o intestino grosso distal, pelo menos até o cólon sigmóide. Resulta obviamente econômico e para os pacientes cômodo, que a avaliação e o tratamento sejam realizados em uma única consulta, seja em consultório ou em hospital. Depois de excluir, através da retossigmoidoscopia flexível, outras causas de hemorragia, como podem ser pólipos, carcinoma, enfermidade intestinal inflamatória, divertículos e angiodisplasias, certificando-se o examinador que o sangramento retal é devido exclusivamente à doença hemorroidária, é conveniente tratá-la neste mesmo momento inicial, sempre que se disponha de um método terapêutico seguro e indolor. Numerosos estudos ${ }^{1,2}$ têm comprovado que o método da Fotocoagulação com Infravermelho é indolor, seguro e eficaz para todas as Hemorróidas não prolapsadas e para algumas com pequenos prolapsos.

Trabalho realizado na Clínica de Proctologia do Maranhão

Recebido em 30/03/2006

Aceito para publicação em 25/04/2006 
A Fotocoagulação Infravermelha foi descrita por Nath e col. (1977) para a coagulação de vasos sangrantes, evitando a aderência dos tecidos ao cautério, algo normalmente obtido com a diatermia. Este método foi adaptado ao tratamento eletivo da Doença Hemorroidária por Neiger ( 1979).

$\mathrm{O}$ tratamento fundamenta-se no emprego de pulsos de 1,5 segundos de irradiação infravermelha para aumentar a temperatura tecidual até $100^{\circ} \mathrm{C}$ e produzir uma área de coagulação proteica de $3 \mathrm{~mm}$ de diâmetro por $3 \mathrm{~mm}$ de profundidade. $\mathrm{O}$ tecido queimado reage da mesma forma que o tecido criodestuído ou que o tecido estrangulado por um anel de ligadura. A resposta tecidual ocorre no plano existente entre o tecido necrótico e o preservado.

Após 10 a 14 dias, o tecido necrótico separase deixando uma úlcera recoberta por tecido de granulação. O índice de reepitelização varia de acordo com o tamanho da úlcera, mas em geral, se completa ao cabo de 4 semanas.

\section{METODOLOGIA}

\section{Pacientes}

O presente trabalho corresponde à observação e análise de resultados da aplicação de Fotocoagulação Infravermelha, já na primeira consulta, em 250 pacientes consultados na Clínica de Proctologia do Maranhão, por apresentarem proctorragia supostamente devida a Hemorróidas, no período compreendido entre março/2001 a outubro/2004. A idade variou entre 20 a 58 anos, sendo a média de 39 anos. Quanto ao sexo, houve predominância do masculino (120) sobre o feminino (80), correspondendo a 60 e $40 \%$ respectivamente.

Foram excluídos 50 pacientes $(20 \%)$ por apresentarem crises de hemorragia retal vermelho vivo, devido ao achado à retossigmoidoscopia flexível, com abrangência até a flexura esplênica, das seguintes patologias.

Tumores retais e sigmoidianos

Divertículos sigmoidianos

Pólipos

Enfermidade Intestinal Inflamatória

Angiodisplasias
$10(20 \%)$

$20(40 \%)$

$10(20 \%)$

$8(16 \%)$

$2(4 \%)$

\section{EQUIPAMENTO E TÉCNICA DA APLICAÇÃO}

A fonte da radiação infravermelha é uma lâmpada halogena de $15 \mathrm{~V}$ tipo Woolfram cujos raios são refletidos por um refletor folheado a ouro e dirigidos a uma haste de cristal de quartzo. Através de um condutor luminico, esta radiação é levada até a ponte da haste, a qual é recoberta por uma capa polímera, que evita a aderência da sonda aos tecidos. $\mathrm{O}$ aparelho possui um temporizador que permite regular o tempo de emissão da radiação. Diferente do que ocorre com a crioterapia hemorroidária, há muito pouca irradiação energética, pois o tempo de emissão dos raios é muito curto, não sendo, portanto, necessária a utilização de um anuscópio termo resistente.

Todos os pacientes, com exceção dos que apresentam repleção de fezes no reto, podem ser imediatamente tratados já na primeira consulta. A técnica é realizada com o paciente em decúbito lateral esquerdo (posição de Sims), sendo que a duração do tratamento não costuma passar de 2 minutos.

Com a base do mamilo projetada para o interior da extremidade do anuscópio, a ponta da sonda é aplicada exercendo-se uma suave pressão sobre a mucosa da base da hemorróida interna, assim como também lateralmente, perfazendo um total de 3 pontos de fotocoagulação para cada plexo hemorroidário. O pulso da irradiação é automaticamente controlado. Se não houver um contato total entre a ponta da sonda e a mucosa, o muco ou material fecal que eventualmente se interpuser entre a sonda e a mucosa, aderirá àquela e o aumento da temperatura no material acolado abrirá um orifício sob a extremidade do polímero. É muito importante que a ponta esteja em contato completo com a mucosa. Ao se retirar a ponta da sonda da mucosa, pode-se imediatamente ver uma pequena área de coagulação. Todo o procedimento na base de cada mamilo leva menos de 30 segundos para ser realizado.

Todos os pacientes são orientados a aumentarem a ingesta de fibras nos dias subseqüentes, assim como também notificarem qualquer ocorrência de dor mais intensa não controlada através de analgésicos comuns. Os pacientes são também avisados da possibilidade de ocorrerem pequenos sangramentos retais na primeira semana após a realização do trata- 
mento, devido à queda da escara nos pontos fotocoagulados.

Recomenda-se uma visita de revisão após 8 semanas do tratamento, sendo os pacientes sempre alertados para notificar qualquer ocorrência de sangramento após este período.

\section{RESULTADOS}

Estudos de longa duração a respeito deste método ainda não estão disponíveis. Leicster e Col. (1981) ${ }^{8}$ relatam ter seguido 25 pacientes por 3 meses, sendo que 21 deles ou estavam assintomáticos ou referiram ter melhorado de seus sintomas. Apenas um paciente relatou dor de grande intensidade e seis descreveram sangramento após o tratamento. Nenhum outro efeito colateral foi relatado e todas as ulcerações estavam completamente cicatrizadas após 6 semanas.

Ambrose e Col. (1983) ${ }^{10}$ trataram pacientes por meio da fotocoagulação como parte de um estudo que a comparava com a ligadura elástica. Os pacientes eram portadores de Hemorróidas de primeiro grau $(70 \%)$ e de segundo grau (30\%). A fotocoagulação associou-se a um número menor de efeitos colaterais do que a ligadura elástica, embora os resultados clínicos tenham sido semelhantes para os dois procedimentos.

Templeton e Col. (1983) ${ }^{10}$ realizaram um estudo muito semelhante em Belfast com destinação randômica dos pacientes ou para o grupo de ligadura elástica ou para o da fotocoagulação. 66 pacientes foram tratados pela fotocoagulação infravermelha e observaram resultados satisfatórios em $85 \%$ deles. Houve menor complicação com a fotocoagulação, sendo concluído que é um método simples, rápido e eficiente.

No presente trabalho, dos 200 pacientes tratados, 158 (79\%) precisaram de uma única sessão de tratamento. 42 pacientes $(21 \%)$ regressaram após 8 semanas, sendo que $25(59 \%)$ por apresentarem hemorragia continuada, foram submetidos a uma segunda sessão de fotocoagulação. 17 pacientes (41\%) necessitaram mais de duas sessões de tratamento. Do total de 42 pacientes que necessitaram de mais de uma sessão de fotocoagulação, 12 (28\%) tiveram que ser submetidos a Hemorroidectomia, em virtude da falha do tratamento. Comparando-se com o número total dos pacientes tratados por este método, ou seja, 200 pacientes, tivemos uma percentagem de insucesso em torno de 6\%. Em todos os pacientes, nos quais o tratamento não surtiu os resultados desejados, não existiam causas associadas da proctorragia.

Nenhum dos nossos pacientes necessitou ser hospitalizado ou transfundido por causa de hemorragia secundária ao procedimento, assim como também não foi observado nenhum tipo de complicação urinária ou infecciosa. Não tivemos nenhum caso de estenose.

\section{DISCUSSÃO}

Os aspectos mais importantes a serem considerados no presente trabalho são a baixa morbimortalidade causada por este procedimento e a quase completa ausência de dor. Se não são aplicadas mais que 3 coagulações em cada plexo hemorroidário, parece que o procedimento está completamente livre de complicações. O método de seguimento não é perfeito, podendo ocorrer a possibilidade de algum paciente, não satisfeito, procurar outro especialista.

Posto que a proctorragia é um sintoma intermitente e a razão principal para realizar uma consulta, e o temor de que a hemorragia possa ter um significado grave, é possível que, uma vez dissipado este temor, os pacientes negligenciem seu tratamento. Também é possível que o próprio procedimento de investigação, incluindo a ligeira dilatação que se produz pela passagem do retossigmoidoscópio ou anuscópio, possa controlar a hemorragia.

A principal conclusão que se pode extrair deste estudo é que uma vez realizadas as explorações pertinentes aos pacientes que apresentam uma hemorragia retal de sangue vermelho vivo, se não se detecta outra causa desta, um procedimento terapêutico rápido e desprovido de inconvenientes, consiste em fotocoagular os plexos hemorroidários principais. Este tratamento é capaz de aliviar a sintomatologia da maioria dos pacientes.

\section{COMPLICAÇÕES}

Durante as três primeiras semanas após o tratamento, muitos pacientes podem apresentar proctorragias ocasionais, não tendo sido registrado nenhum caso de hemorragia vultuosa que necessitasse hospitalização. Dos pacientes especificamente 
interrogados, a maioria manifesta ausência ou dor de intensidade mínima controlada com administração de analgésicos comuns. A dor é avaliada utilizando-se a escala de descritores numéricos e verbais, assim como a escala analógica visual.

A proctoscopia realizada durante a recuperação mostra uma área de necrose circular de diferentes intensidades, porém apesar disto, a cicatrização é satisfatória, sem estenose ou sequelas a longo prazo.

\section{CONCLUSÃO}

Vários estudos comparativos ${ }^{6,10,11}$ têm comprovado que a fotocoagulação com infravermelho ocasiona menos dor que a ligadura elástica ou crioterapia para hemorróidas internas e que, no controle da hemorragia é tanto ou mais eficaz que a escleroterapia por injeção retal submucosa. A maioria dos autores ${ }^{7}$ recomenda 3 aplicações de fotocoagulação ao redor de cada plexo hemorroidário, perfazendo um total de 9 pontos de coagulação, considerando a existência anatômica de 3 plexos hemorroidários, todos tratados em uma única sessão.
Um desconforto transitório durante a aplicação da sonda é comum, porém não é freqüiente ocorrer dor prolongada. A fotocoagulação provavelmente é o método menos doloroso dentre os métodos conservadores do tratamento das hemorróidas. Não é necessária a aplicação de anestesia regional, nem costuma haver necessidade de prescrição de analgésicos orais no período pós procedimento. Se o paciente queixa-se de desconforto contínuo é porque o local escolhido para a aplicação dos raios estava situado muito próximo da linha pectínea.

Como o tecido é apenas superficialmente lesado, a incidência e gravidade de sangramento secundário são extremamente raras, pois a duração da emissão da radiação limita a destruição tecidual a $3 \mathrm{~mm}$ de profundidade, de forma que estruturas mais profundas não podem ser lesadas.

Comparando-se com outras formas de tratamento conservador para hemorróidas internas sangrantes, como a escleroterapia por injeção, o anel elástico e a crioterapia, podemos concluir que a Fotocoagulação Infravermelha é o método mais seguro, rápido e eficaz. $\mathrm{O}$ aparelho não é necessariamente caro e resulta essencial para o tratamento das proctorragias devidas à Doença Hemorroidária Interna.

SUMMARY: A great number of patients are frequently referred for specialist advice because they have experienced rectal bleeding. The bright rectal bleeding usually does not come from anywhere more proximal than the splenic flexure. Complete evaluation should include a flexible sigmoidoscopy. During evaluation many patients are found to have Internal Haemorrhoids that could well be the source of bleeding. There is always a dilemma: whether or not to reassure the patients that the bleeding is caused by nothing more serious than simple haemorrhoids or whether to consider that the reference of the diagnosis included an obligation to treat. I have pursued a policy of treating all patients referred for assessment of the cause of bleeding when the only source of bleeding seems to be piles. They have the base of their internal haemorrhoids coagulated with the photocoagulator. Of 250 patients attended at the Clinic of Proctology of Maranhão because of rectal bleeding, 200 were treated with minimal morbidity and needed just one treatment stage. It is proposed that photocoagulation is such a simple trouble-free form of treatment that it can safely be applied at the end of any evaluation for bright red rectal bleeding and that such policy enables the majority of those referred patients to be managed in a single visit.

Key words: Anal bleeding, Haemorrhoids, Photo Coagulation

\section{REFERÊNCIAS BIBLIOGRÁFICAS}

1. Ambrose MS, Morris D, Alexsander Williams J, Keighley MRB (1983). A randomized trial of Photocoagulation or injection sclerotherapy for the treatment of first and second degree haemorrhoids. Dis. Colon Rectum 28: 238-240

2. Buls JG, Goldeberg SM (1978) Modern management of haemorrhoids. Surg Clin North Am 58: 469-478
3. Clarn CG, Eiles G. Goligher JC (1967). Results of conservative treatment of internal haemorrhoids. Br. Med J 2: 12

4. Dercker H, Hjorth M, Norryd L, Tramberg KG (1973). Comparison of results obtained with different methods of treatment of internal haemorrhoids. Acta Chir Scared 139: 742

5. Gehamy RA, Weakley FL (1974). Internal haemorrhoidectomy by elastic ligation. Dis Colon Rectum 17: 347-353 
6. Jones CB, Schofield PF (1974). A comparative study of the methods of treatment for haemorrhoids. Proc R Soc Méd 67: $51-53$

7. Kaufman HD (1976) Out-patient treatment of haemorrhoids, Br J Surg 63: 462-463

8. Leicster RJ, Nicholls RJ, Mann CV (1981) Infra red coagulation: a new treatment for haemorrhoids. Dis Colon Rectum 24: 602

9. Neiger A (1979) Haemorrhoids in everyday practice. Proctology 2: 22

10. Templeton JL, Spence RAJ, Kennedy Th et al (1983) Comparison of Infra red coagulation and rubber band ligation for first and second degree haemorrhoids: a randomized prospective Clinical Trial Br Med J 286: 1387

11. Walker AJ, Leivster RN, Nicholls RJ, Mann CV (1990). A prospective study of infra red coagulation, injection and rubber band ligation in the treatment of haemorrhoids. Int $\mathrm{J}$ Colorrectal DIS 5: 113-116
12. Weinstein SJ, Rypins EB, Houck J, Thrower S (1987) Single Session treatment for bleeding haemorrhoids. Surg Gynecol Obstet 165: 479-482

\section{Endereço para correspondência:} JOSÉ RIBAMAR BALDEZ

Clínica de Proctologia do Maranhão Avenida Colares Moreira, $555-5^{\circ}$ andar Edifício Renascença Medical Center - Renascença II 65.075-441 - São Luís (MA)

Tel: (98) 3268-2929 / 3217-4458

E-mail: cpm@elo.com.br 